\title{
Relevance of d-D interactions on neutron and tritium production in IFMIF-EVEDA accelerator prototype
}

\author{
A. Mayoral* ${ }^{*}$ J. Sanz, P. Sauvan, D. López, M. García, F. Ogando \\ Departamento de ingeniería Energética, UNED, 28040 Madird, Spain \\ Instituto de Fusión Nuclear, UPM, 28006 Madrid, Spain
}

\begin{abstract}
A B S T R A C T
In the IFMIF-EVEDA accelerator prototype, deuterium is implanted in the components due to beam losses and in the beam dump, where the beam is stopped. The interaction of the deuterons with the deuterium previously implanted leads to the production of neutrons and tritium, which are important issues for radioprotection and safety analysis. A methodology to assess these production pathways in more realistic approach has been developed. The new tools and their main achievement are: (i) an "effective diffusivity coefficient" (deduced from available experimental data) that enables simulation of the diffusion phase, and (ii) the MCUNED code (able to handle deuteron transport libraries) allows to simulate the transport-slowdown of deuteron/tritium (to get the concentration profiles) and the neutron/tritium productions from $\mathrm{d}-\mathrm{Cu}$ and $\mathrm{d}-\mathrm{D}$ for up to $9 \mathrm{MeV}$ incident deuteron. The results with/without theses tools are presented and their effect on the relevance of $d-D$ sources versus $d-C u$ is evaluated.
\end{abstract}

\section{Introduction}

The JFM]F-EVEDA accelerator will be a $9 \mathrm{MeV}, 125 \mathrm{~mA} C W$ deuteron accelerator, identical to the low energy section of one of the [FMIF accelerators. It will be tested to verify the JFMIF design before launching its construction. This paper proposes a methodology to assess the neutron and tritium sources for radjation protection and safety analysis of this facility.

The following description of the neutrons and tritium production phases can be helpful to focus the issue: (i) deuteron losses from the beam are implanted in the accelerator components and all the remaining deuterons in the beam stop (BS), (ii) the implanted deuterium is transported by diffusion through the material, (iii) neutrons and tritium are produced by interactions of deuterons from the beam with the deuterium previously implanted (d-D reaction) and with the copper material of the accelerator (d-Cu reaction), and (iv) the tritium is thermalized, then transported by diffusion.

The coupled use of the SRIM2008 [1]/MCNPX [2] and TMAP7 [3] codes allows the simulation of these phases. However, some difficulties have to be solved for the IFMIF-EVEDA operating conditions, including: ( $\mathrm{i}$ ) how to determine the deuterium concentration profile inside the copper lattice due to the lack of transport coefficient data for diffusion simulation, (ii) the built-in nuclear models included in MCNPX code (and others as PHITS code $[4,5,6]$ ) do not allow to predict with reasonable accuracy neither the neutron/ tritium production from $\mathrm{d}-\mathrm{Cu}$ and $\mathrm{d}-\mathrm{D}$ reactions for incident deuteron energy up to $9 \mathrm{MeV}$ nor the transport-slowdown unti] implantation of deuterium/tritium (needed to get the concentration profile).

The methodology proposed to solve these difficulties is discussed in Section 2. This methodology is applied to significant zones of the facility and the results are presented and commented in Sections 3 and 4 respectively.

\section{Methodology}

\subsection{Diffision cransport methodology (thermalized deuterons and tritons)}

Traditionally and regardless the irradiation conditions, for d-D neutron production assessment in cooper the "deuterium saturation density" $\left(C_{\text {sat }}=1.7 \times 10^{28} \mathrm{D} / \mathrm{m}^{3}\right)$ is used as a conservative value for deuterium concentration at $293 \mathrm{~K}$ target temperature [7,8]. Actually, this value corresponds to the stationary concentration reached in irradiation conditions $\left(60-85 \mathrm{~mA} / \mathrm{cm}^{2}\right)$ [9] very different from those expected in the IFMIF-EVEDA facility (maximum $0.0158 \mathrm{~mA} / \mathrm{cm}^{2}$ ) [10]. The overestimated results related to the systematically use of the $C_{\text {sat }}$ have been reported by different authors $[7,8,11]$. Even though the assessment of neutron and tritium production from the d-D reaction using the $C_{\text {sat }}$ is a conservative estimation useful for radiation protection issues, we consider necessacy an approach able to predict a more realistic deuterium concentration profile for the JFMIF-EVEDA facility design. 
The TMAP7 code is able to simulate the diffusion transport by concentration gradient forces towards the front and back faces of the structure, including the trapping sites effect (impurities, structural damage, hydrides and bubbles) where the energy required for migration is greater than that required for ordinary diffusion. The accuracy of the TMAP7 simulation depends on the availability of the suitable value for each transport coefficient on specific operation conditions: diffusivity, recombination, trap density, trap energy, etc.

However in the collected data of the literature [12,13] for copper material there are not coefficients for traps, and the temperature range (470-1200 $\mathrm{K}$ ) does not include the IFMIF-EVEDA range (293-373 K). Therefore, in the simulation the trap effect is not considered and is performed out of the suitable temperature range.

Other information sources to get data for the simulation are those from the experimental reports of the bibliography. Fukui et al. reported that traps and temperature are the main parameters determining the stationary hydrogen concentration during irradiation of copper (density, size and energy of traps depends on three parameters: fluence, energy beam and target temperature) [14]. A rise in the energy beam increases the deep of the damage zone and the type and the energy of the traps. The damage in the lattice increases when the fluence rises and the target temperature decreases. Panigrahi et al. suggest that the diffusion of deuterium in cooper during implantation at room temperature is via vacancy mediated migration of deuterium trapped in vacancies $(30 \mathrm{keV}$, $0.025 \mathrm{~mA} / \mathrm{cm}^{2}, 293 \mathrm{~K}, 1.1 \times 10^{19} \mathrm{D} / \mathrm{cm}^{2}$ ) [15]. Anderl et al. reported a trapped-delayed diffusion by beam-induced defects $(3 \mathrm{keV}$, $1.14 \mathrm{~mA} / \mathrm{cm}^{2}, 710-785 \mathrm{~K}, 5.1 \times 10^{19} \mathrm{D} / \mathrm{cm}^{2}$ ) [16]. They proposed a new parameter, called "effective diffusivity" $\left(D_{\mathrm{et}}(T)\right)$. This parameter includes the trap effects (structural damage, bubbles, etc.) on the migration of deuterium. Fig. 1 shows $D_{\mathrm{ef}}(T)$ versus temperature from these experiments (yellow, triangle and square). The trapping effect can be noticed by comparing with the coefficients from the collected data of Reiter et al. [12], $D(T)$, (discontinuous blue line).

It is remarkable how the values proposed by Anderl et al. for temperatures from 710 to $785 \mathrm{~K}$ fit reasonably (by a straight line when plotted on a logarithmic scale versus $1000 / T(K)$ ) with the values proposed by Panigrahi et al. for $293 \mathrm{~K}$ (continuous green line in Fig. 1) [15]. The use of this correlation for $D_{\mathrm{ef}}(T)$ for IFMIF-EVEDA operation conditions $\left(1.0 \times 10^{-10}-0.0158 \mathrm{~mA} / \mathrm{cm}^{2}, 0.1-9.0 \mathrm{MeV}\right.$, 293-785 K) allows overcoming the lack of data for the simulations. However, it is necessary to analyze the reliability of the simulations taking into account that the operation conditions of the original experiments are very different than those of the IFMIF-EVEDA. panigrahi et al. [15] proposed a $D_{\mathrm{ef}}(T)$ constant for fluences up to $1.1 \times 10^{19} \mathrm{D} / \mathrm{cm}^{2}$ and Anderl et al. [16] reported on steady-state condition for the fluence range of his experience $\left(1.0 \times 10^{19}\right.$ $5.1 \times 10^{19} \mathrm{D} / \mathrm{cm}^{2}$ ). For IFMIF-EVEDA operational conditions [10], the time needed to reach this fluence is greater than 200 days in all zones except in the BS and in the first $250 \mathrm{~cm}$ of the RFQ (sections 1 and 2 . The RFQ has been divided in 10 sections, of equal length for calculation purposes). No problems are expected in the BS due to the high temperature [14] but the fluence effect in sections 1 and 2 (ambient temperature, but low energy beam) could be underestimated. Regarding the other parameters, both incident deuteron energy and angle, can be important variables in determining the energy and spatial distribution of traps. However we have no information, so these effects have not been taken into account.

Then, to check the correlation proposed for $D_{\text {er }}(T)$ the deuterium spatial concentration has been computed for the experimental conditions of $C_{\text {sat }}\left(60-85 \mathrm{~mA} / \mathrm{cm}^{2}, 0.08-0.120 \mathrm{MeV}, 293 \mathrm{~K}\right)$ [9]. The small graph in the Fig. 1 includes information regarding to this study. The blue and green lines are the deuterium concentration profile for these experiments from TMAP7 simulations using the proposed $D_{\text {ef }}$ and the red line is the $C_{\text {sat }}$ value. This work shows that the proposed approach allows a reasonable agreement with the experiments.

The work performed in this paper includes the neutron and tritium production for the IFMIF-EVEDA facility calculated using the three following approaches for the deuterium concentration: (i) $C_{\text {sat }}$ (ii) collected data with trap effect neglected and not taking into account the suitable temperature range and (iii) the correlation for the $D_{\text {eft }}(T)$.

Finally, an improvement in the TMAP7 code was used to transport simultaneously the two species (deuterium and produced tritium) in a reliable way, in order to simulate the diffusion transport towards the vacuum pump and the cooling water.

\subsection{Monte Carlo transport methodology (energetic deuterons and tritons)}

A reliable assessment of the neutron production induced by deuterons as well as the simulation of the deuterium/tritium implantation phase are not possible due to the poor agreement

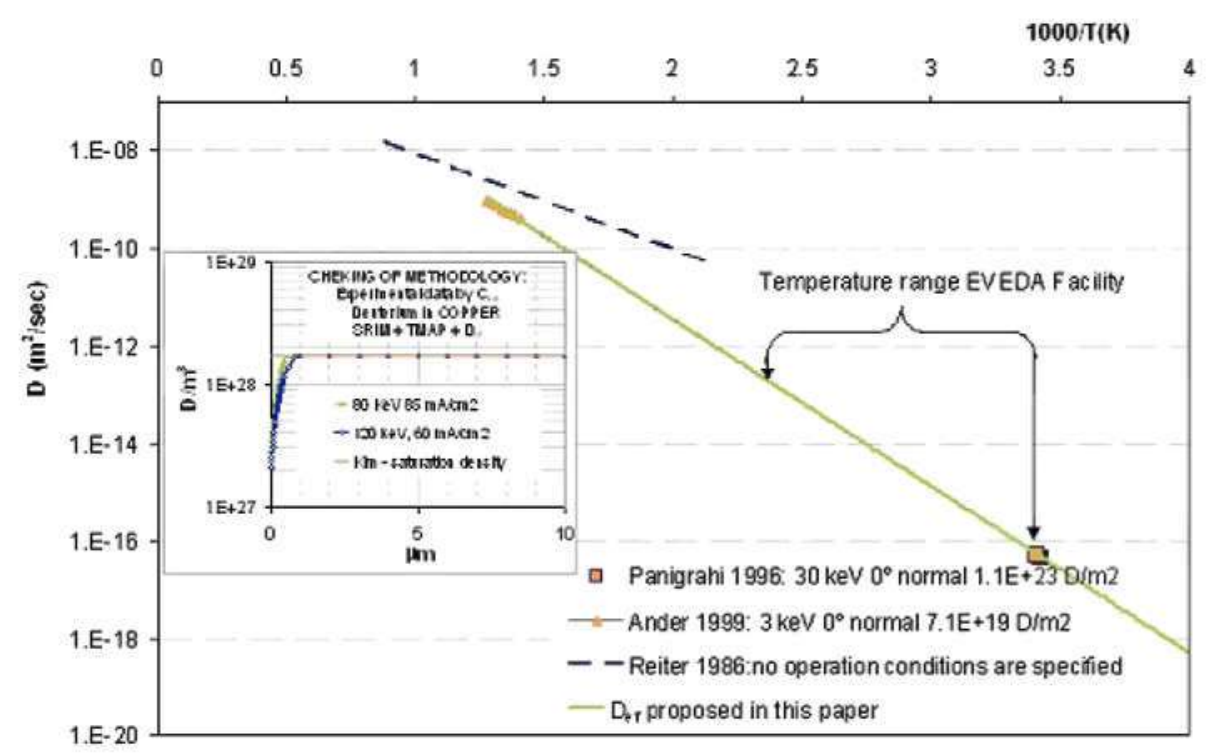

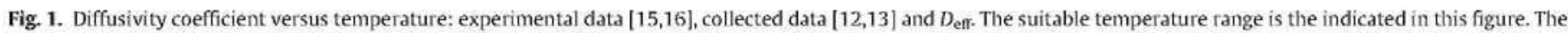
small figure shows the deuterium concentration $\left(\mathrm{D} / \mathrm{m}^{3}\right)$ for a depth up to $10 \mu \mathrm{m}$ in copper from $D_{\text {ef }}$ approach simulation by $C_{\text {sat }}$ experimental conditions $[8,9]$. 
between the XS derived from the built-in nuclear models included in MCNPX and experimental data for deuteron interactions with energies up to $9 \mathrm{MeV}[5,6]$. An example of the severe underestimation when comparing the experimental data with the Incl4, Isabel and LAQGSM built-in MCNPX models is showed in Fig. 2 for $(\mathrm{d}, 2 \mathrm{n})$ reaction (only for this reaction experimental data is available for neutron production from the $\mathrm{d}-\mathrm{Cu}$ interaction).

Therefore it was necessary to: i) search for an external deuteron double differential XS database for neutron/tritium production which agrees reasonably with experimental data in the IFMIF-EVEDA operating range, and ii) to develop a transport code able to handle those external libraries.

The details of both tasks have been described elsewhere: Sanz et al, $[5,17]$ for the XS libraries validated with experimental data for neutron productions from the $\mathrm{d}-\mathrm{Cu}$ reactions, and Sauvan et al. [18] for the new modified MCNPX code named MCUNED.

The neutron and tritium production rates and the concentration profile of implanted tritium were computed using the following procedures: i) MCNPX code using default built-in nuclear model (Isabel/Dressner), ii) MCUNED code with ENDF-VII [19] and TENDL2008 [20] (Talys based [21]) transport libraries for d-D and d-Cu reactions respectively. Fig. 2 shows a reasonable agreement for Talys with experiments, slightly conservative, for Cu65(d,2n) and a good agreement between ENDF and experimental data for $\mathrm{D}(\mathrm{d}, \mathrm{n})$ reaction.

The simulation of the deuteron losses along the accelerator line was provided by Comunian (García [6]) and the details of the division are described in $[6,10]$. The Fig. 3 includes information relative to the simulations (energy of the deuterons losses, the deuteron losses, the incident angle respect the normal and the temperature of the intercepting material). For all the accelerator elements (RFQ MS, DTL and BS) copper has been considered as intercepting material.

The flow chart (codes, XS libraries and diffusion coefficients) proposed to a more realistic approach to the neutron and tritium productions is showed in Fig. 4.

\section{Results}

The proposed methodology has been applied to assess the following issues for the IFMIF-EVEDA facility.

(A) The deuterium concentration profile obtained using the following three approaches: (i) the most conservative approximation: $C_{\text {sat }}$ (ii) a less conservative approximation: assuming trapping is neglected, (iii) the approximation proposed in this paper: $D_{\text {ef }}(T)$ correlation taking into account trap effect. The Fig. 3 shows the deuterium concentration profile for EVEDA-IFMIF sections by $D_{\mathrm{ef}}$ approach. The difference between $C_{\text {sat }}$ and $D_{\text {ef }}$ approaches are close to two orders of magnitude in sections 4 and 5 of RFQ, in the MS and in the BS.

(B) The time needed to achieve a stationary deuterium concentration profile (therefore a steady-state in neutron and tritium production from d-D reactions). This time will be reduced when the temperature or the current density increases. In the case of section 1 of the RFQ and in the BS, $6 \mathrm{~h}$ is enough to reach the steady-state but 10 days are needed for section 10 (the stationary conditions are reached when the deuterium concentration at $20 \mu \mathrm{m}$ depth-higher than deuterium penetration- is $90 \%$ of the value reached for 365 days of continuous irradiation).

(C) The neutron production from d-Cu and from d-D (taking into account the three approaches for the deuterium concentration assessment) by MCUNED and MCNPX codes. The results for neutron production in sections 1 and 10 of the accelerator and in the BS using the different approaches are presented in Table 1. These sections 1 and 10 are the first and the last of the RFQ (length of each section: $1.25 \mathrm{~m}$ ) and are the more significant for the conclusions of this work (Fig. 3). The higher deuterium concentration for low and high energies in the accelerator line, respectively $(0.1$ and $5 \mathrm{MeV}, 158$ and $4 \mathrm{~mA} / \mathrm{m}^{2}$, incident beam $85^{\circ}$ with respect to the normal, $293 \mathrm{~K}$ ) have been obtained. The BS is object of high current density and the higher energy of the facility $\left(158 \mathrm{~mA} / \mathrm{m}^{2}, 9 \mathrm{MeV}\right.$, incident beam $86.57^{\circ}$ with respect to the normal, $393 \mathrm{~K}$ ). The MCNPX code is not able to compute in a reliable way the neutron production in the RFQ (energy up to $5 \mathrm{MeV}$ ) and the assessment in BS (energy up to $9 \mathrm{MeV}$ ) is slightly underestimated.

(D) The tritium production by d-D and d-Cu reactions (only for $D_{\mathrm{ef}}(T)+$ MCUNED procedure). The tritium produced is mainly due to the d-D reaction (95\%). The cross sections of tritium and neutron production in $\mathrm{d}-\mathrm{D}$ reaction are similar for energies up to $9 \mathrm{MeV}$. Therefore we assume that the effect of the approach used to compute the deuterium concentration (saturation density, trapping neglected or effective diffusivity) on the tritium production is the same than that observed on the neutron production. The tritium inventory in the BS and the amount that reaches the cooling water given by TMAP7 simulations for 365 days of continuous
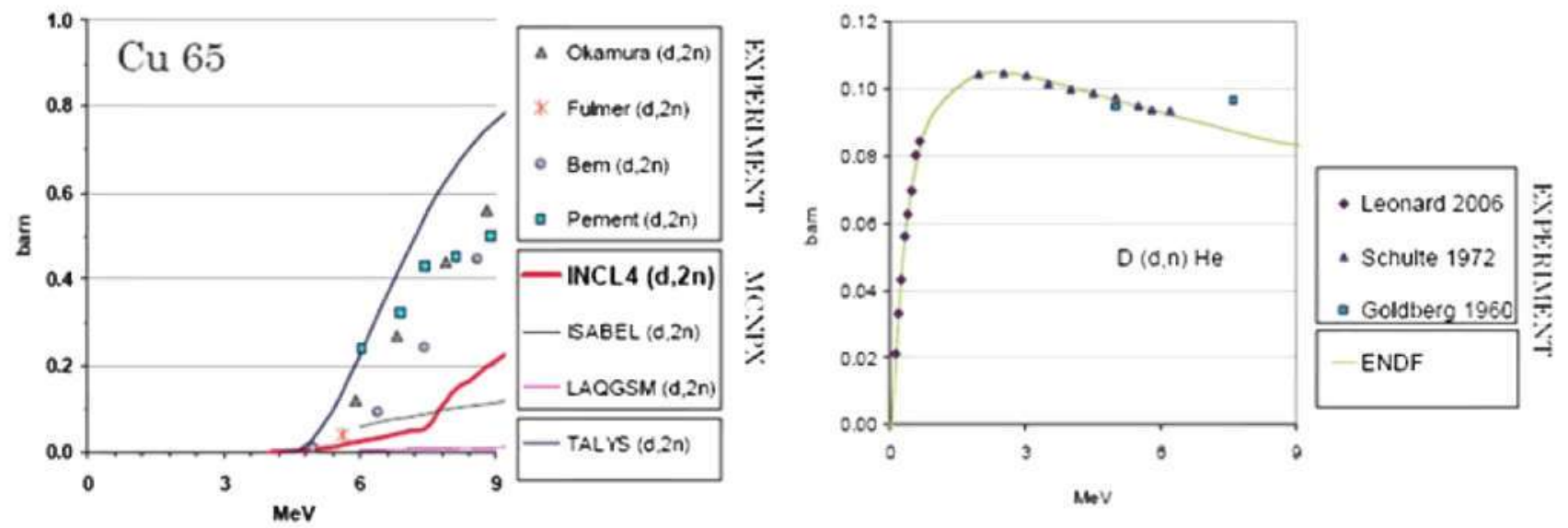

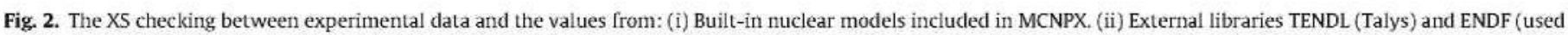
by MCUNED code). 


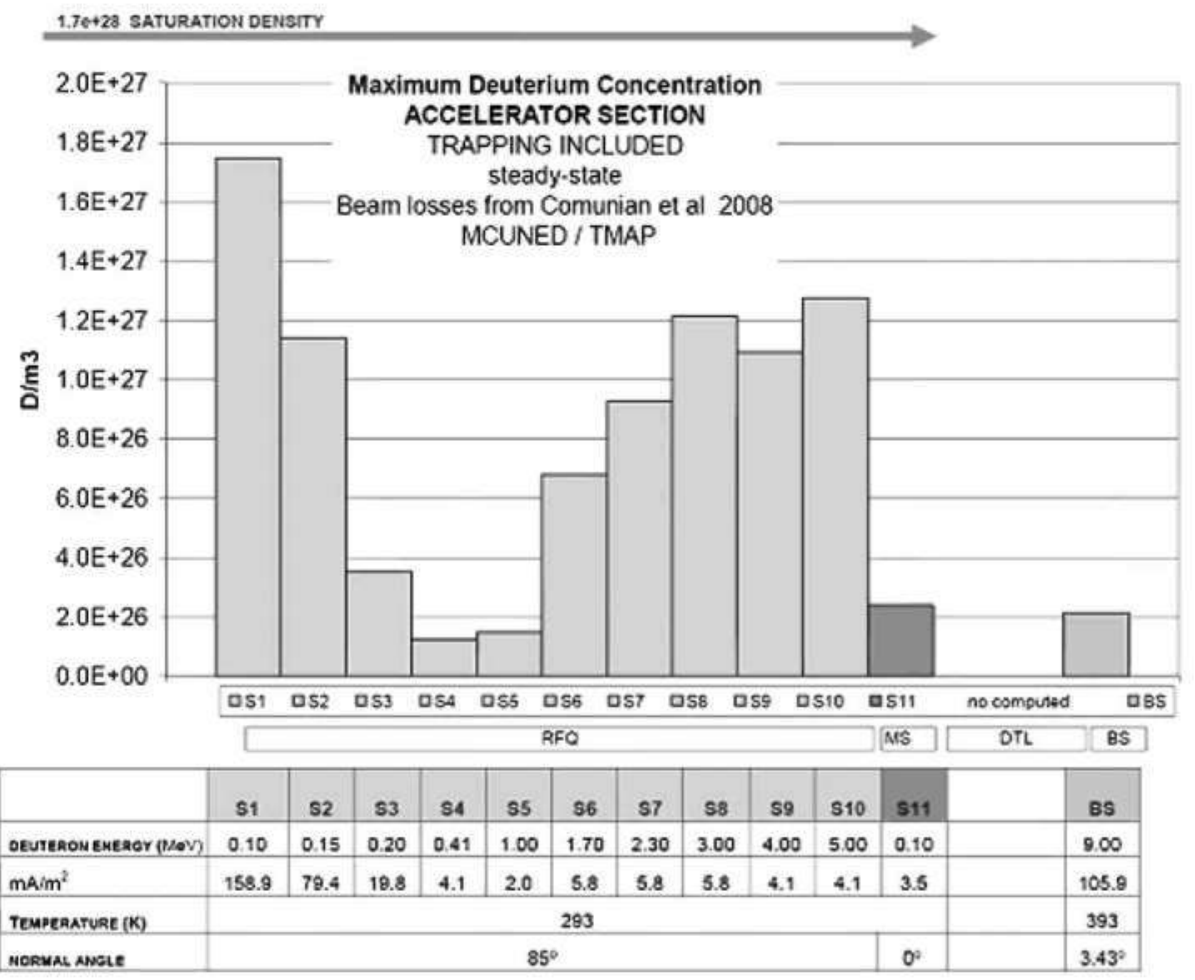

Fig. 3. Maximum deuterium concentration for EVEDA-IFMIF accelerator sections using $D_{\text {ef }}$ approach.

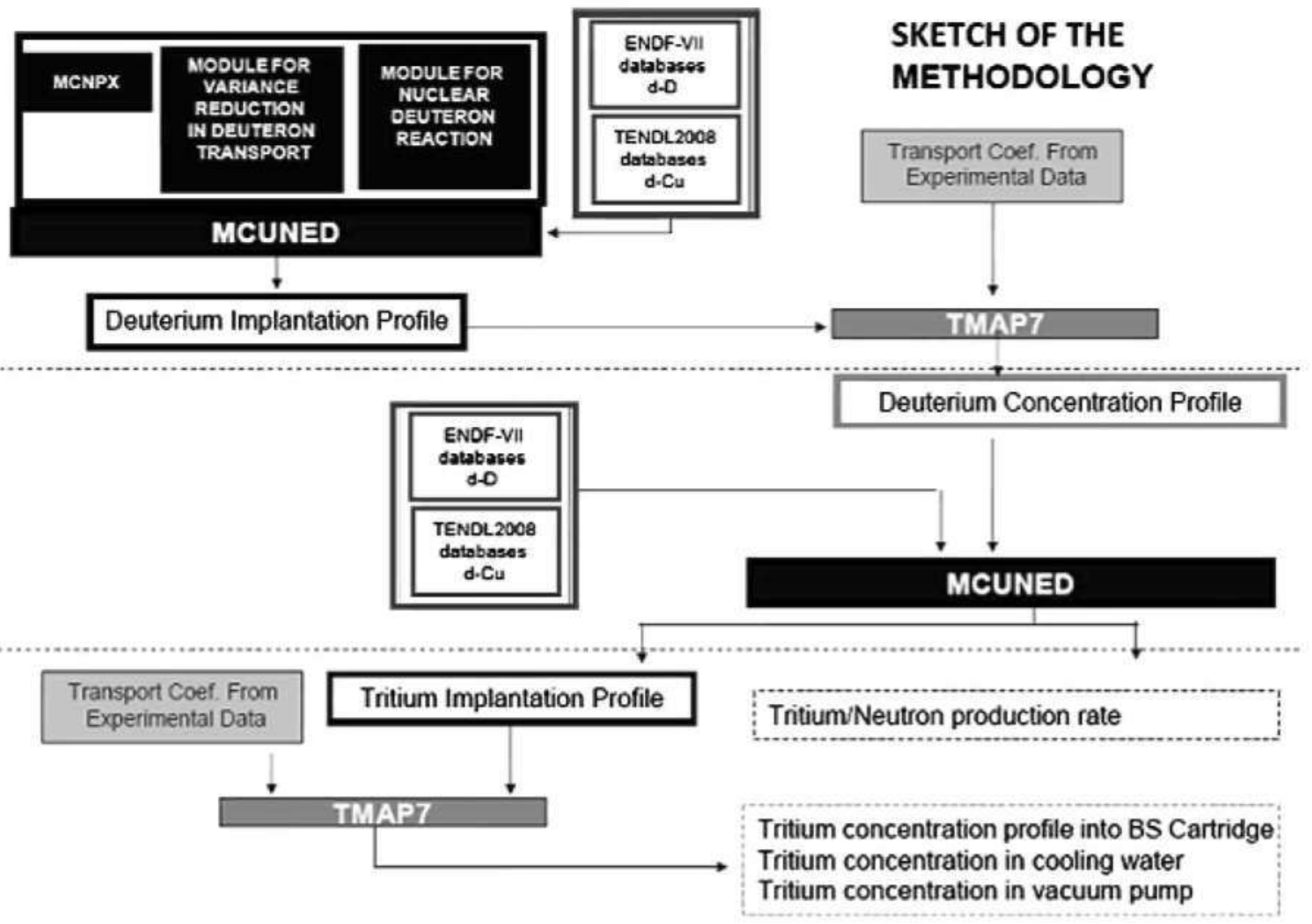

Fig. 4. Diagram of the proposed methodology. 
Table 1

Neutron production from d-D and d-Cu reactions for stationary state computed by: (i) MCUNED and external clata libraries and (ii) MCNPX.

\begin{tabular}{|c|c|c|c|c|c|c|}
\hline \multirow[t]{4}{*}{ zone } & \multirow[t]{4}{*}{ Hypothesis to compute the D concentration profile } & \multicolumn{5}{|c|}{ NEUTRON SOURCE SIMULATION APPROACH } \\
\hline & & \multicolumn{3}{|l|}{ neutron/sec. } & \multicolumn{2}{|l|}{ MCNPX } \\
\hline & & \multicolumn{3}{|l|}{ MCUNED } & \multicolumn{2}{|l|}{ MCNPX } \\
\hline & & d-D (ENDF-VII) & d-Cu (TENDL2008) & Total & d-D & d-CU (ISABEL/DRESSNER) \\
\hline \multicolumn{7}{|l|}{ RFQ } \\
\hline Section 1 & Trapping included & $1.6 \mathrm{E}+07$ & $6.4 \mathrm{E}+01$ & $1.6 \mathrm{E}+07$ & 0 & 0 \\
\hline $0.1 \mathrm{MeV}$ & Trapping neglected & $3.7 \mathrm{E}+05$ & & $3.7 \mathrm{E}+05$ & & \\
\hline $2.9 \mathrm{E}+16 \mathrm{~d} / \mathrm{s}$ & Saturation density & $2.7 \mathrm{E}+08$ & & $2.7 \mathrm{E}+08$ & & \\
\hline \multicolumn{7}{|l|}{ RFQ } \\
\hline Section 10 & Trapping neglected & $4.1 \mathrm{E}+08$ & $5.2 \mathrm{E}+10$ & $5.2 \mathrm{E}+10$ & 0 & 0 \\
\hline $5 \mathrm{MeV}$ & Trapping included & $2.7 \mathrm{E}+06$ & & $5.2 \mathrm{E}+10$ & & \\
\hline $3.9 \mathrm{E}+16 \mathrm{~d} / \mathrm{s}$ & Saturation density & $8.7 \mathrm{E}+09$ & & $6.0 \mathrm{E}+10$ & & \\
\hline BS & Trapping neglected & $1.1 \mathrm{E}+11$ & $4.8 \mathrm{E}+14$ & $4.8 \mathrm{E}+14$ & $1.1+\mathrm{E} 11$ & $1.3+\mathrm{E} 14$ \\
\hline $9 \mathrm{MeV}$ & Trapping included & $3.9 \mathrm{E}+10$ & & $4.8 \mathrm{E}+14$ & & \\
\hline $7.8 \mathrm{E}+17 \mathrm{~d} / \mathrm{s}$ & Saturation density & $1.6 \mathrm{E}+13$ & & $5.0 \mathrm{E}+14$ & & \\
\hline
\end{tabular}

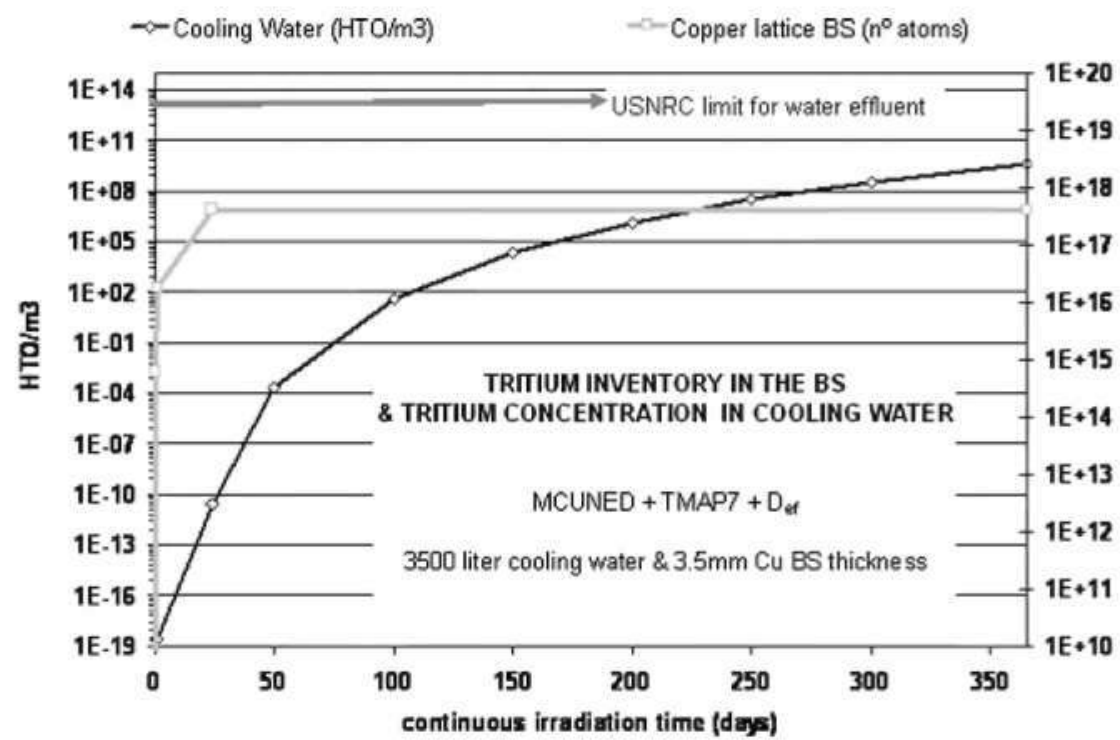

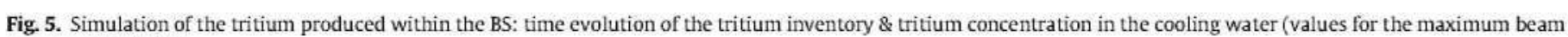
power, $2.1 \mathrm{E}+11 \mathrm{t} / \mathrm{s})$.

irradiation are shown in Fig. 5. This figure shows that the tritium concentration in the BS cooling water is lower than the USNRC limit value in effluents [22].

\section{Conclusions}

A new methodology to assess the neutron and tritium production using a more realistic approach for application to the IFMIFEVEDA facility has been developed. This methodology solves the hard overestimation of the deuterium concentration using the traditional saturation density approach and the lack of a transport code able to compute the neutron/tritium production from deuteron interaction in cooper for energy up to $5 \mathrm{MeV}$.

The "effective diffusivity" correlation for deuterium in copper is proposed to overcome the lack of data on trap coefficients needed to perform the diffusion simulation. The use of this correlation allows a more reliable profile of the deuterium concentration in copper compared with the constant value of the "saturation density", $C_{\text {sat }}$ traditionally used for neutron production assessment. The deuterium concentration in copper using this procedure is up to two orders of magnitude lower than those obtained using the traditional tools.
The coupled use of both tools ( $D_{\mathrm{ef}}$ and MCUNED code) allows a more reliable estimation of the neutron/tritium production from d$\mathrm{D}$ and $\mathrm{d}-\mathrm{Cu}$ reactions at EVEDA-IFMIF facility. For energy up to $5 \mathrm{MeV}$, only MCUNED code allows to compute the neutron production. For energy range from 5 to $9 \mathrm{MeV}$ the neutron/tritium production from d-D decreased up to two orders of magnitude comparing with the traditional approach. Therefore, the procedure proposed in this paper allows a more reasonable evaluation on the neutron/tritium production from d-D and from d-Cu in the different sections of the facility. The results of the simulations show that the neutron production from d-D respect to $d-C u$ reactions varies hardly: from 6 orders of magnitude higher in section 1 to 2 orders lower in section 10 of the RFQ and 3 orders lower in BS.

The study about the time needed to reach the steady-state for neutron/tritium production rates from $d-D$ reactions concludes that this time is reduced when the temperature or the current density is increased. For EVEDA-IFMIF facility this time is in the range of few hours to 10 days. This time is very important in those accelerator sections where the d-D neutron production cannot be neglected compared with the neutron produced from d-Cu.

The tritium (mainly produced by the $d-D$ reaction) that reaches the cooling water of the BS has a concentration in water lower than 
the limit value reported by the US Nuclear Regulatory Commission for water in effluents. Nevertheless this value must be added to other sources of tritium for the final evaluation about the relevance of the tritium concentration in this effluent.

Finally, is important to be mentioned that the availability of more accurate XS libraries would be able to get a more reliable results in the neutron/tritium production. Regarding $D_{\text {er }}(T)$ more effort is necessary in this way, since it was deduced only from two experimental reports for operational range very different than the expected in EVEDA-IFMIF facility. Though temperature and fluence effects on traps were taken into account, in sections 1 and 2 of the RFQ where high fluence is aggravated by low temperature, we have no enough reliability on the deuterium concentration profiles obtained. Moreover the information about the effect of deuteron energy and incident angle on the traps is not enough, and it was not taken into account. Future experiments for fluence, energy and angle data in the range of EVEDA-JFMJF facility, will be helpfu] to get a more realistic value of the $D_{\mathrm{e}}(T)$.

\section{Acknowledgment}

This work has been partially supported by Association EURATOM/CIEMAT for Fusion (AEC) within the framework of JFM]F-EVEDA Project in the Broader. Approach Agreement. It has been also partially supported by Plan Nacional de ] + D + [ (20082011) Fusion Nuclear, ENE2008-06403-C06-02, MEC, Spain.

\section{References}

[1] J.F. Ziegler, M.D. Ziegler, J.P. Biersack, SRIM 2008 Code. The Stopping and Range of lons in Matter, 2008.

[2] D.B. Pelowitz (Ed.), MCNPX User's Manual, Version 2.5.0, LA-CP-05-0369 (2005). User's Manual, Version 2.6.0, LA-CP-07-1473. JS. Hendricks et al.: MCNPX 2.6.0 Extensions, LA-UR-08-2216 <http:/fmcmpx.lanl.gov>, 2008.
|3| G.R. longhurst, TMAP7; 1D. Transport of Tritium and Other Gas in Materials via Diffusion Model, Longhurst, INNEL. TMAP7 Users Manual, INEEL/EXT-0402352. INNEL, 2004.

[4] Koji Niita et al., Radiat. Meas. 41 (2006) 1080-1090.

|5| J. Sanz, M. Galcía, F. Ogando, A. Mayoral, D. López, P. Sauvan, B. Blañas, Fusion Sci. Technol. 56 (1) (2009) 273-280.

[6] M. García, J. Sanz, P. Sauvan, F. Ogando, D. López, V. Blideanu, A. Mayoral, C. Moreno. Nucl. Teclinol. 168 (1) (2009) 132-138.

[7] T.T.C. Jones, S.J. Cox, A. Emmanoulidis, M.J. Loughlin, Nucl. Fusion 46 (2006) 352-359.

[8| J. Kim, Nucl, Instrum, Methods 145 (1977) 9-17.

[9] J. Kim, Nucl. Technol. 44 (1979) 315-321.

[10] B. Bratias et al., Prelimary design of JFMIF-EVEDA Beam Dump, Draft. April, 2009.

[11] M. Fumelli, P. Bayetti, R. Becherer, F. Bottiglioni, M. Desmonds, F. Jequier, J Pamela, P. Raimbault, F.P.G. Valcks, Sci. Instrum. 57 (7) (1986) 1266-1273.

[12] F. Reiter, K.S. Forcey, G. Gervasini, A compilation of tritium-material interaction palameters in fusion reactor materials. Joint Research Centre. ISPRA Site Institute for Safety Technology. Commission of the European Communities. EUR 15217EN, 1993.

[13] T.J. Dolan, RA. Anderl, Assessment of Database for lnteraction of Tritium with ITER Plasma Facing Materials. EGG-FSP-11348 ITER/93/USTTUSA=10, 1994.

[14] M. Fukui, R. Sakamoto, K. Alaki, T. Fujiwara, T. Muroga, N. Yoshida. J. Nucl. Mater. 220-222 (1995) 810-814.

[15] B. Panigrahi, K.G.M. Nair, K. Krishan, Bull. Mater. Sci. 19 (1) (1996) 61-72.

[16] R.A. Anderl, M.R. Hankins, G.R. Longhurst, R.J. Pawelko. J. Nucl. Mater, 266-269 (1999) 761-765.

[17] V. Blideanu, M. Garcia, Ph. Joyer, D. Lopez, A. Mayoral, F. Ogando, J. Sanz, P. Sauvan. Deuteron cross section evaluation for safety and ladioprotection calculations of IFMIF, in: Proceedings, Sapporo (lapan), 7-12 September, 2009.

[18] P. Sauvan. J. Sanz. F. Ogando. Nucl. Inst. Methods Phys. Res., A 614 (2010) 323330.

[19] M.B. Chadwick, P. Oblozinsliy. M. Herman, et al., Nucl. Data Sheets 107 (2006) 2931-3060.

[20] A.J. Koning, D. Rochman, Consistent Talys-based Evaluated Nuclear Data library including covariance data (TENDL2008 <http://www.talys.eu/tendl2008/s).

[21] A.J. Koning. 5. Hilaire, M.C. DUujvestijn, "TALY5-1.0", in: Proc, of the lnt Conference on Nuclear Data for Science and Technology - ND2007, April 2227, 2007 Nice France editors O. Bersillon, F. Gunsing, E. Bauge, R. Jacgmin, S. Leray (Eds.). EDP Sciences, 2008. pp. 211-214.

[22] United Stated Nuclear Regulatory Commission recommendations. (USNRC Table XXX4) <http://www.nic.gov/reading-lm/doc-collections/cfi/parto2o/ appb/s. 